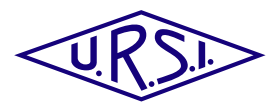

\title{
Flexible Multi-standard Digital Front-End for LPWA Technologies
}

\author{
Patrick Savelli $^{(1)}$, Vincent Savaux $^{(1)}$, Pauline Desnos $^{(1)}$, Ali Zeineddine ${ }^{(1)}$, Matthieu Kanj $^{(1)}$, and Christophe Delacourt ${ }^{(1)}$ \\ (1) $\mathrm{b}<>$ com, Rennes, France
}

\begin{abstract}
This paper proposes a flexible multi-standard digital frontend (DFE) hardware architecture designed for three main low-power wide-area (LPWA) technologies: LoRa, Sigfox, and narrowband-Internet of things (NB-IoT). We demonstrate the feasibility of an unified DFE architecture that fits the requirements of these LPWA technologies. The proposed DFE architecture has been implemented on a Spartan-6 field programmable gate array (FPGA) within a base station receiver, and tested using a universal software radio peripheral (USRP) based platform. We show that the expected performance can be achieved with low hardware complexity, in terms of memory and logic requirements.
\end{abstract}

\section{Introduction}

The development of the Internet of things (IoT) market has led to a great diversity of wireless connectivity solutions. This paper addresses the low-power wide-area (LPWA) solutions, targeting IoT networks with coverage in the kilometer order, using low-power end devices having a battery life autonomy of multiple years. Various LPWA technologies and standards have emerged, with different air interfaces : LoRa, Sigfox, NB-IoT, LTE-M, DASH7, Weightless, NB-Fi, RPMA, MYTHINGS, and others $[1,2,3]$. In this context where multiple options are possible, there is a need for technical components that are flexible enough to support different technologies. In this paper, we focus on the main LPWA technologies that have been widely adopted and deployed by operators, as part of large ecosystems [4]: LoRa, Sigfox and NB-IoT

The LoRa technology introduced by Semtech uses a chirpbased patented modulation scheme, while the network can be deployed by any third-party entity based on the LoRaWAN open specifications [5] maintained by the LoRa Alliance ${ }^{1}$. Sigfox technology proposed by Sigfox S.A. also uses the unlicensed spectrum, and is based on the differential binary phase-shift keying (D-BPSK) and Gaussian frequency shift keying (GFSK) modulation schemes. Moreover, the network deployment of this technology is controlled by Sigfox. NB-IoT, initially published in Release 13 of the third generation partnership project (3GPP) organization ${ }^{2}$, is based on cellular mobile network, using resources within frequency bands allocated to LTE (inband mode), or dedicated to NB-IoT (standalone mode).

\footnotetext{
${ }^{1}$ https://https://lora-alliance.org/

${ }^{2}$ https://www.3gpp.org/news-events/1785-nb_iot_ complete
}

This diversity increases the LPWA network deployment cost in case each technology is independently deployed.

To solve the problems of a heterogeneous network, and to efficiently exploit virtualization techniques, the interoperability between these LPWA technologies should be maximized. On the radio front-end level, this is achieved by providing LPWA gateways that are capable of supporting the largest number of technologies. This type of gateways requires a digital front-end (DFE) implementation that provides the flexibility needed to meet the requirements of these different technologies.

This paper proposes a generic approach to build a flexible multi-standard DFE for LPWA technologies. A focus on the hardware implementation of the DFE is presented. A common architecture for the three considered LPWA solutions is proposed, whilst respecting the constraints imposed by their specifications.

The rest of the paper is organized as follows. In section 2, the flexible DFE architecture is presented based on the requirements of the different LPWA technologies. Section 3 presents the hardware implementation of this flexible DFE. Section 4 concludes this work.

\section{Multi-standard DFE design approach}

\subsection{Overview}

A preliminary analysis phase of the three protocols allowed us to design the DFE around three blocks shown in Fig. 1, and described as follows:

- Rate adaptation: the sampled signal at the output of the analog to digital converter (ADC) is sub-sampled from sampling frequency $f_{0}$ (ADC frequency) to frequency $f_{1}$, with $f_{1} \leq f_{0}$. A very efficient and low-complexity rate adaptation is implemented based on [6,7].

- Filtering: A low-pass finite impulse response (FIR) filter operating at frequency $f_{1}$, allows to reject neighbouring interferers and blocking signals, as well as performing a pre-equalization in the band-pass area. Since the computational cost of the filtering step is directly related to the length of the filter (i.e. its number of taps), it is designed as short as possible while respecting the requirements. We used our own FIR filter design tool $^{3}$ to generate equiripple filters [8].

\footnotetext{
${ }^{3}$ available at https://wirelesslibrary.labs.b-com.com/ FIRfilterdesigner/
} 


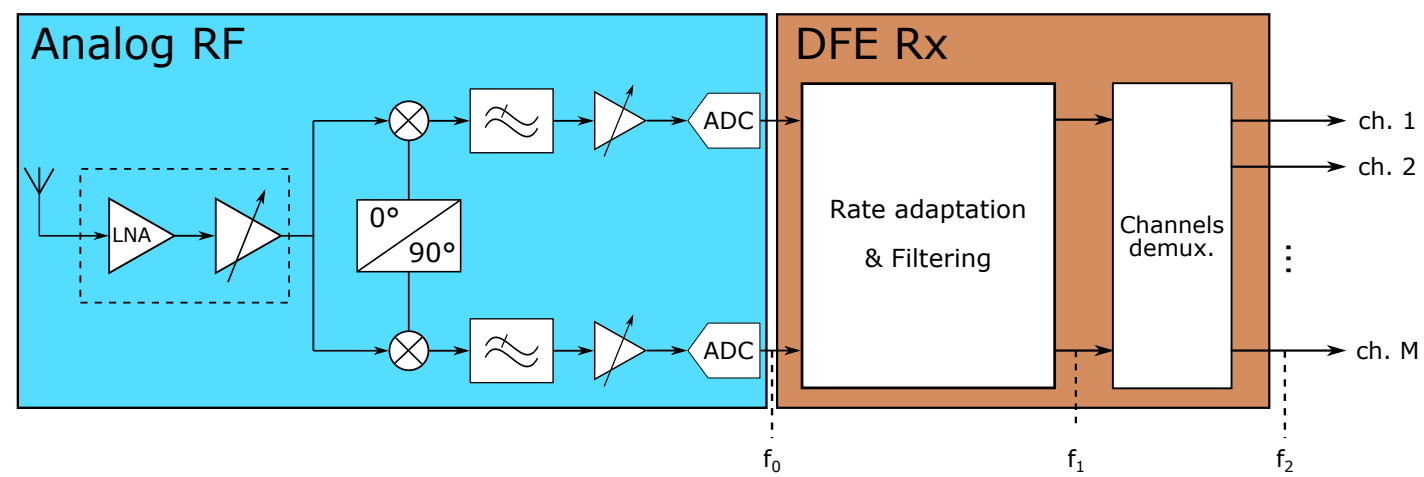

Figure 1. General DFE block diagram, including rate adaptation, filtering, and channels demultiplexing (demux.).

- Channels demultiplexing: the aim of the channels demultiplexing block is to split the whole band into different channels, e.g. channels of $125 \mathrm{kHz}$ in LoRa (i.e. $f_{2} \geq 125$ $\mathrm{kHz}$ ) or of $180 \mathrm{kHz}$ in NB-IoT (i.e. $f_{2} \geq 180 \mathrm{kHz}$ ). It must be noted that there is no default channel split defined in Sigfox, so we propose to apply several channel demultiplexing blocks in parallel, using the same input signal with a different frequency shift, so as to use a similar architecture.

These blocks are common to the three technologies, and only require specific parameters to be adapted to each one of these. It then allows for rapid hardware developments of flexible DFEs. Fig. 2 shows an example of frequency response for the rate adaptation, the low-pass filter, and the combination of the latter (referred to as "global"). In this example, the DFE is adapted to the reception of NB-IoT within an LTE band, considering a $5 \mathrm{MHz}$ bandwidth. The $\mathrm{ADC}$ frequency is set to $f_{0}=60.8 \mathrm{MHz}$ and the output frequency of the rate adaptation and filtering stage is $f_{1}=7.68 \mathrm{MHz}$, corresponding to the sampling frequency of the $5 \mathrm{MHz}$ LTE band. The filter has been designed for a $100 \mathrm{~dB}$ rejection and a ripple below \pm 1 $\mathrm{dB}$. Furthermore, it can be observed that the filter applies an equalization in the pass-band to counterbalance the attenuation due to the rate adaptation block.

\subsection{Design constraints}

We provide in this section the list of RF performance parameters that impact the DFE design for the targeted air interfaces.

LoRa: LoRa networks are operated in unlicensed bands using a proprietary physical layer (PHY) designed by Semtech. The LoRa PHY main characteristics are listed in Table 1. The European industrial, scientific and medical (ISM) frequency band used for LoRa operation is indicated for the sake of example, but other unlicensed frequency bands are also used according to the geographical area [9].

Based on these characteristics, we have derived the design parameters of our DFE for the LoRa mode of operation as indicated in Table 2. The provided parameters have been

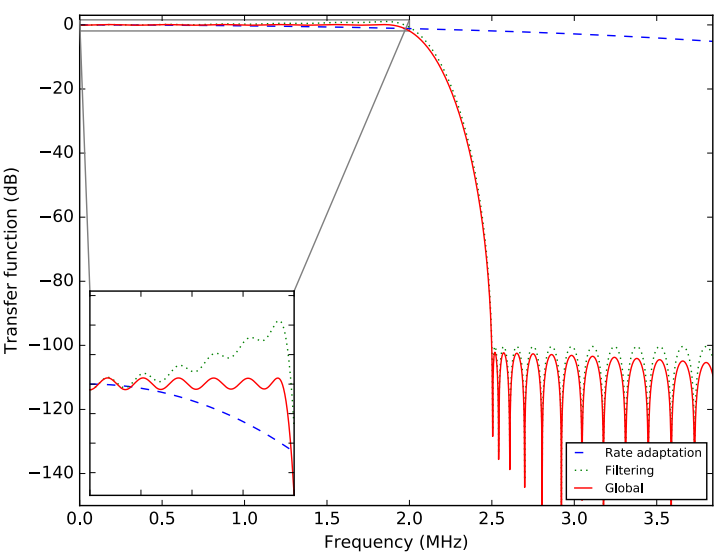

Figure 2. Filter shapes at each step and global filtering. Note that FIR filter compensates the rate adaptation attenuation in pass band.

carefully adjusted to achieve high channel selectivity, assuming the parallel reception of $8 \mathrm{LoRa} \mathrm{RF}$ channels with $200 \mathrm{kHz}$ channel raster. The output rate of the DFE in this case is $250 \mathrm{kSamples} / \mathrm{s}$, which corresponds to a $1 \mathrm{MB} / \mathrm{s}$ per channel data rate when using 16 bits for each of the I and Q paths.

Sigfox: Sigfox has released their proprietary radio specifications in [10], including the air interface description and the different regional parameters. Table 3 summarizes the radio parameters of the European region. As indicated in this table, the available frequency range for uplink and downlink transmissions spans over $192 \mathrm{kHz}$ in both directions. Within this bandwidth, guard bands are reserved at the lower and upper parts of the band to account for the inaccuracies in the reference frequency at the end node (20ppm assumed) and at the network gateway (1.62ppm assumed) sides. The usable frequency band when considering these guard bands is reduced to $154.462 \mathrm{kHz}$, centered at $868.13 \mathrm{MHz}$. For the sake of clarity, let us consider a usable frequency band of $150 \mathrm{kHz}$ in the rest of this article.

It is also important to note that the channel bandwidth occupied by the Sigfox signal $(100 \mathrm{~Hz})$ is low compared to the channel frequency inaccuracies of the transmitter and receiver ends. For this reason, the Sigfox gateway receiver cannot assume a 
Table 1. LoRa PHY characteristics

\begin{tabular}{|c|c|}
\hline & Europe \\
\hline Frequency band & $863-870 \mathrm{MHz}$ \\
\hline Duplex mode & half duplex \\
\hline Channel bandwidth & $125 \mathrm{kHz}$ \\
\hline Channel raster & $200 \mathrm{kHz}$ \\
\hline Number of channels & 3 mandatory, typically 8 \\
\hline Gateway Tx power & $20 \mathrm{dBm}$ \\
\hline Modulation & Chirp Spread Spectrum \\
\hline Spreading Factor & $7-12$ \\
\hline Coding rate & $4 / 5,4 / 6,4 / 7,4 / 8$ \\
\hline
\end{tabular}

Table 2. DFE configuration for LoRa, 8 channels

\begin{tabular}{|c|c|}
\hline \multicolumn{2}{|c|}{ DFE design parameters } \\
\hline ADCs output Rate $f_{0}$ & $60.8 \mathrm{MHz}$ \\
\hline Rate adapt. output rate $f_{1}$ & $1.6 \mathrm{MHz}$ \\
\hline $\begin{array}{c}\text { Channels demultiplexing } \\
\text { output rate } f_{2}\end{array}$ & $\begin{array}{c}250 \mathrm{kSamples} / \mathrm{s} \\
\text { per channel }\end{array}$ \\
\hline number of filter taps & 500 \\
\hline \hline \multicolumn{2}{|c|}{ Achieved channel selectivity } \\
\hline carrier offset & rejection \\
\hline \hline $85 \mathrm{kHz}$ & $75 \mathrm{~dB}$ \\
\hline
\end{tabular}

fixed channel raster, and must be able to demodulate an uplink modulated signal present at any frequency within the usable bandwidth. In addition, the average RF channel frequency drift observed during a transmission is as high as $\pm 20 \mathrm{~Hz} / \mathrm{sec}$ due to the reference frequency source drift, and the typical burst duration exceeds 1 seconds. This constraint needs to be addressed when designing the filtering and channel demultiplexing in the DFE

In order to fulfill the above constraints, our proposal is to consider $300 \mathrm{~Hz}$ wide channels every $200 \mathrm{~Hz}$, using two parallel filtering and channels demultiplexing entities. Each parallel entity processes a different version of the received signal shifted by a $200 \mathrm{~Hz}$ offset. In total, there are 750 channels at the output of the DFE to cover the $150 \mathrm{kHz}$ band. The DFE parameters set to implement this Sigfox configuration are provided in Table 4. Note that at the DFE output, the channel bandwidth is $300 \mathrm{~Hz}$, so it is required to further process the output of the DFE to demodulate the 100 bauds D-BPSK signal.

NB-IoT: NB-IoT is a cellular LPWA technology introduced in the Release 13 of the 3 GPP specifications. In this contribution, we target the NB-IoT inband operation mode, where some LTE resource blocks of $180 \mathrm{kHz}$ bandwidth are allocated to NB-IoT. For instance, four specific resource blocks are defined when NB-IoT is transmitted in the $5 \mathrm{MHz}$ LTE band. Our proposal for the NB-IoT operation mode is to use the DFE for the extraction of $180 \mathrm{kHz}$ wide physical resource blocks (PRB) within the LTE band. Tables 5 and 6 summarize the NB-IoT constraints and the corresponding configuration, respectively, considering the LTE $5 \mathrm{MHz}$ bandwidth, and inband mode.
Table 3. Sigfox PHY characteristics

\begin{tabular}{|c|c|}
\hline & Europe \\
\hline Frequency band & $\begin{array}{c}868.034-868.226 \mathrm{MHz} \text { uplink } \\
869.429-869.621 \mathrm{MHz} \text { downlink }\end{array}$ \\
\hline Duplex mode & FDD \\
\hline Channel bandwidth & $100 \mathrm{~Hz}$ uplink \& downlink \\
\hline Modulation & D-BPSK uplink \\
& GFSK downlink \\
\hline
\end{tabular}

Table 4. DFE configuration for Sigfox

\begin{tabular}{|c|c|}
\hline \multicolumn{2}{|c|}{ DFE design parameters } \\
\hline ADCs output Rate $f_{0}$ & $60 \mathrm{MHz}$ \\
\hline Rate adapt. output rate $f_{1}$ & $150 \mathrm{kHz}$ \\
\hline $\begin{array}{c}\text { Channels demultiplexing } \\
\text { output rate } f_{2}\end{array}$ & $\begin{array}{l}\text { 400Samples/s } \\
\text { per channel }\end{array}$ \\
\hline number of filter taps & 24000 \\
\hline \multicolumn{2}{|c|}{ Achieved channel selectivity } \\
\hline carrier offset & rejection \\
\hline $\pm 200 \mathrm{~Hz}$ & $60 \mathrm{~dB}$ \\
\hline
\end{tabular}

\section{Hardware Implementation}

\subsection{Test platform}

Our gateway experimentation platform is composed of an offthe-shelf NI USRP B205mini board. This software defined radio (SDR) platform contains the $\mathrm{RF}$ transceiver and an Xilinx Spartan-6 FPGA with a $83.3 \times 50.8 \times 8.4 \mathrm{~mm}$ form factor. The DFE function has been implemented in the user programmable FPGA. The receiver also includes, prior to the B205mini RF receiver, an analog front-end board of our own design, that includes a low noise amplifier (LNA) achieving $30 \mathrm{~dB}$ gain with $3 \mathrm{~dB}$ noise figure (NF) in the $868 \mathrm{MHz}$ ISM band and the LTE band 8 . The functional and performance tests are done using IoT (LoRa, Sigfox or NB-IoT) commercial devices.

\subsection{FPGA implementation and results}

Table 7 summarizes the Rx DFE hardware resources utilization for each of the targeted IoT standards. Four key resources are detailed: registers used to save temporary results and variables, look-up tables (LUTs) that determine the combinatorial behaviour, memories (RAMs) to store parameters such as the filter taps, and digital signal processors (DSPs) that are specialized units for multiplication/addition computation.

This table shows that any of the LoRa, Sigfox or NB-IoT DFEs fits in the small cost-effective B205mini board. The different technologies use similar resources, except for the RAM requirement that is much higher for the Sigfox mode. This is mainly due to the number of channels to be processed in parallel, requiring a large amount of filter taps. The RAM requirement is also higher in case of NB-IoT compared to LoRa. The worst case in NB-IoT inband mode is indeed the $20 \mathrm{MHz}$ LTE bandwidth case, where 18 PRBs are dedicated to NB-IoT and need to be extracted in parallel. This impacts the number of filter 
Table 5. NB-IoT PHY characteristics (inband mode)

\begin{tabular}{|c|c|}
\hline Frequency bands & same as LTE \\
\hline Duplex mode & $\begin{array}{c}\text { Half duplex } \\
\text { FDD } \\
\text { (release 15) }\end{array}$ \\
\hline Channel bandwidth & $180 \mathrm{kHz}$ \\
\hline Waveform & SC-FDMA (uplink) \\
& OFDMA (downlink) \\
\hline Modulation & $\pi / 2$-BPSK, $\pi / 2$-QPSK, QPSK \\
\hline Subcarrier spacing & $3.75 \mathrm{kHz}, 15 \mathrm{kHz}$ \\
\hline
\end{tabular}

Table 6. DFE configuration for NB-IoT inband mode, $5 \mathrm{MHz}$ bandwidth (25 PRBs)

\begin{tabular}{|c|c|}
\hline \multicolumn{2}{|c|}{ DFE design parameters } \\
\hline ADCs output Rate $f_{0}$ & $60.8 \mathrm{MHz}$ \\
\hline Rate adapt. output rate $f_{1}$ & $7.68 \mathrm{kHz}$ \\
\hline $\begin{array}{c}\text { Channels demultiplexing } \\
\text { output rate } f_{2}\end{array}$ & $\begin{array}{c}240.10^{3} \text { Samples/s } \\
\text { per channel }\end{array}$ \\
\hline number of filter taps & 256 \\
\hline \multicolumn{2}{|c|}{ Achieved channel selectivity } \\
\hline carrier offset & rejection \\
\hline $\pm 200 \mathrm{~Hz}$ & $600 \mathrm{~dB}$ \\
\hline
\end{tabular}

taps that need to be stored within the block. In case of LoRa, the considered number of parallel channels is set to 8 , hence the lower memory needs. Moreover, it must be emphasized that a small (cost-effective) FPGA has been used for our test, allowing for the embedding of at most two technologies on the same board. However, the three technologies could be easily embedded on a larger board, allowing for a true multi-standard DFE.

The integration of the DFE within the complete network infrastructure has been initiated, and preliminary measurements have been conducted. Fig. 3 shows some of the sensitivity measurements results obtained for the LoRa case. The curves represent the block error rate as a function of the input signal level, for different spreading factor configurations (SF).

\section{Conclusion}

In this paper, we present a flexible multi-standard digital DFE for the main LPWA technologies: LoRa, Sigfox and NB-IoT. The solution is based on three hardware subsystems that can be configured and tuned according to the targeted technology: rate adaptation, filtering and channel demultiplexing. In the future, we plan to assess and further improve the full RF receiver performance for the three technologies, and particularly immunity to adjacent signals and blockers. We are also working on the integration of hardware detection blocks dedicated to the above mentioned technologies with encouraging preliminary results.

\section{References}

[1] Keysight Technologies, "Internet of Things (IoT) (Poster),"
Table 7. FPGA DFE complexity figures

\begin{tabular}{|l|cccc|}
\hline & Registers & LUTs & RAM & DSP \\
\hline Available & 184304 & 92152 & 268 & 180 \\
\hline LoRa & 12055 & 10162 & 24 & 68 \\
Sigfox & 12282 & 11019 & 153 & 68 \\
NB-IoT & 13585 & 10936 & 48 & 74 \\
\hline
\end{tabular}

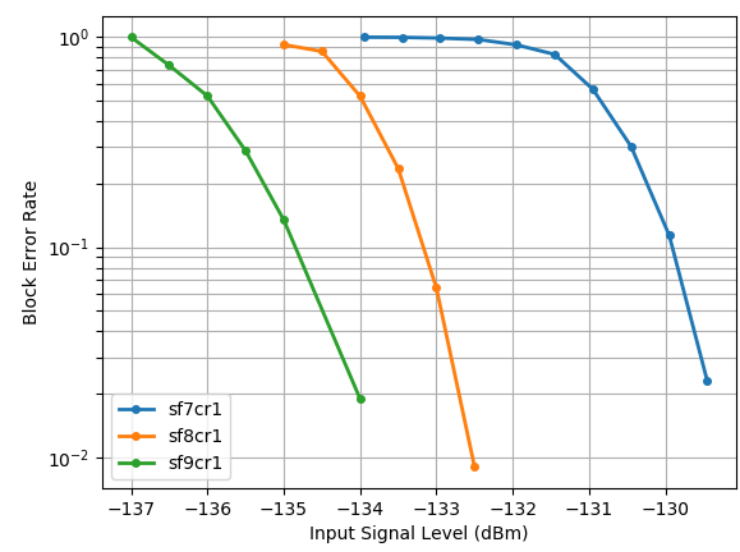

Figure 3. Sensitivity preliminary measurements for LoRa system

May 2016. [Online]. Available: https://www.keysight.com/fr/ en/assets/7018-05035/posters/5992-1217.pdf

[2] W. Ayoub, A. Samhat, F. Nouvel, M. Mroue, and J.-C. Prévotet, "Internet of Mobile Things: Overview of LoRaWAN,DASH7, and NB-IoT in LPWANs standards andSupported Mobility," in proc. of ICT'18, June 2018, pp. 1561 - 1581.

[3] K. Mekki, E. Bajic, F. Chaxel, and F. Meyer, "A comparative study of LPWAN technologies for large-scale IoT deployment," ICT Express, vol. 5, no. 1, pp. 1-7, March 2019.

[4] IoT Analytics, "LPWAN Market Report 2018-2023," September 2018. [Online]. Available: https://iot-analytics.com/product/ lpwan-market-report-2018-2023/

[5] "Lorawan ${ }^{\mathrm{TM}}$ specification," LoRa Alliance Technical Committee, October 2017, ver. 1.1

[6] A. Zeineddine, S. Paquelet, M. Kanj, C. Moy, A. Nafkha, and P. Jezequel, "Reconfigurable newton structure for sample rate conversion," in 2018 IEEE Global Conference on Signal and Information Processing (GlobalSIP). IEEE, 2018, pp. 271275.

[7] A. Zeineddine, S. Paquelet, A. Nafkha, P.-Y. Jezequel, and C. Moy, "Efficient arbitrary sample rate conversion for multistandard digital front-ends," in 2019 17th International IEEE NEW Circuits and Systems Conference (NEWCAS), jun 2019.

[8] S. Paquelet and V. Savaux, "On the symmetry of FIR filter with linear phase," Elsevier Digital Signal Processing, vol. 81, no. 10, pp. 57 -60, October 2018.

[9] "Lorawan®regional parameters," LoRa Alliance Technical Committee, November 2019, ver. 2-1.0.0.

[10] "Sigfox connected objects: Radio specifications," sigfox, November 2019, rev. 1.4. [Online]. Available: https: //build.sigfox.com/sigfox-device-radio-specifications 